\title{
Both the serum AFP test and AFP/GPC3/ SALL4 immunohistochemistry are beneficial for predicting the prognosis of gastric adenocarcinoma
}

Bingzhi Wang ${ }^{1 \dagger}$, Yibin Xie ${ }^{2 \dagger}$, Li Zheng ${ }^{3}$, Xiaohao Zheng ${ }^{2}$, Jia Gao ${ }^{4}$, Xiuyun Liu' ${ }^{1}$, Yanling Yuan ${ }^{1}$, Zhuo Li', Ning Lu' and Liyan Xue ${ }^{1 *}$

\begin{abstract}
Background: Both gastric adenocarcinoma with primitive enterocyte phenotype (GAPEP) (including hepatoid adenocarcinoma) and alpha-fetoprotein (AFP)-producing gastric adenocarcinoma have poor prognoses. However, the value of the serum AFP test and AFP/glypican-3 (GPC3)/spalt-like transcription factor 4 (SALL4) immunohistochemistry is still not clear, and these two methods have not yet been thoroughly compared.

Methods: We collected 421 consecutive non-neoadjuvant surgically or endoscopically resected gastric adenocarcinoma patients with serum AFP results before surgery (group A). We divided these cases into serum AFP-high (SAFP-H) and serum AFP-normal (sAFP-N) by serum AFP levels, and into GAPEP (expressing AFP, GPC3, or SALL4) and non-GAPEP (nGAPEP) by AFP/GPC3/SALL4 immunohistochemistry results. We also collected 12 non-resected gastric adenocarcinoma patients with serum AFP $\geq 7 \mathrm{ng} / \mathrm{mL}$ before treatment (group B). We analyzed these patients' clinicopathological characteristics and prognoses.

Results: Seventeen (4.04\%) patients in group A were SAFP-H. These patients were younger and mainly had tubular adenocarcinoma with later $\mathrm{pT}(P=0.014)$ and $\mathrm{pN}(P=0.047)$ categories and more lymphovascular invasion $(P<0.001)$, perineural spread $(P=0.008)$, and metastases or recurrence $(P<0.001)$. For immunohistochemistry, $34(8.08 \%)$ cases were GAPEP, and GAPEP cases also had later pT categories than nGAPEP cases $(P=0.001)$. Most group $B$ patients with elevated serum AFP (especially $>1000 \mathrm{ng} / \mathrm{mL}$ ) had simultaneous metastases, mainly liver metastases. Both the serological method and immunohistochemical method were useful for predicting prognosis ( $A \cup C_{\text {sAFP }}=0.625$, AUC $\mathrm{A} / \mathrm{G} / \mathrm{S}-\mathrm{HC}=0.723, \mathrm{z}$ statistic $=1.726, P=0.084)$. The serum AFP level (especially $>1000 \mathrm{ng} / \mathrm{mL}$ ) is more specific $(100 \%)$, and immunohistochemistry is more sensitive (50\%).
\end{abstract}

Conclusion: Both the serum AFP level and immunohistochemical expression of AFP/GPC3/SALL4 can be used to indicate a poor prognosis for gastric adenocarcinoma.

Keywords: Gastric cancer, Pathology, Biomarker, Metastases

*Correspondence: xuely@cicams.ac.cn

${ }^{\dagger}$ Bingzhi Wang and Yibin Xie have contributed equally

1 Department of Pathology, National Cancer Center/National Clinical Research Center for Cancer/Cancer Hospital, Chinese Academy of Medical Sciences and Peking Union Medical College, Beijing 100021, China Full list of author information is available at the end of the article

\section{Background}

Gastric cancer is one of the most common malignant tumors and a heterogeneous malignant tumor [1]. Although most gastric cancers are adenocarcinomas, their biological behavior and prognosis are significantly 
different. The detection of serum biomarkers is helpful for predicting the prognosis of gastric adenocarcinomas $[2,3]$. One of the most widely studied markers is serum alpha-fetoprotein (sAFP). In 1970, Bourreille et al. proposed the concept of hepatoid adenocarcinoma (HAC) based on morphology and found that this subtype was often accompanied by elevated SAFP and more likely to have liver metastases $[4,5]$. Subsequent studies also found that gastric adenocarcinoma with enteroblastic differentiation (GAED) and yolk-sac tumor-like carcinoma had similar characteristics [6, 7]. Successive studies concluded that regardless of whether there was a special pathological morphology, cases of positive AFP immunohistochemistry or elevated sAFP had a suggestive risk of progression, collectively referred to as "AFP-producing gastric adenocarcinoma" [8, 9]. This concept suggests transformation from morphology to molecular biology. With the application of more immunohistochemical markers, Yamazawa et al. found that AFP, glypican-3 (GPC3) and spalt-like transcription factor 4 (SALL4) immunohistochemistry (A/G/S-IHC) outcomes had similar effects. Patients expressing at least one of these proteins (AFP, GPC3 or SALL4) had a poor prognosis and frequently exhibited liver metastases regardless of morphology. They called this subtype "gastric adenocarcinoma with primitive enterocyte phenotype (GAPEP)" [10].

However, several unresolved problems remain in previous studies. The relationship between GAPEP and SAFP level is not clear, and no studies have compared the SAFP level and A/G/S-IHC results in gastric adenocarcinoma. The cutoff value for sAFP elevation is not uniform, and the specific significance of the degree of SAFP elevation is not clear [11]. In most hospitals, the sAFP test and A/G/S-IHC test are not routine tests for gastric carcinoma patients, though both methods are easy to perform widely.

In this study, we collected gastric adenocarcinoma cases with definite sAFP levels and then analyzed their clinicopathological characteristics and immunohistochemical results of AFP, GPC3, and SALL4. We tried to determine the value of SAFP and A/G/S-IHC in the diagnosis and treatment of gastric adenocarcinoma.

\section{Materials and methods}

\section{Enrollment criteria}

For enrollment, patients must have met the following conditions: admission to the National Cancer Center/ National Clinical Research Center for Cancer/Cancer Hospital, Chinese Academy of Medical Sciences and Peking Union Medical College between December 2016 and December 2018 and without history of germ cell tumors, primary hepatocellular carcinoma or active hepatitis. We also excluded the cases with probable liver cirrhosis according to imaging and blood biochemistry reports. Two groups were established. Group A included 421 cases consecutive non-neoadjuvant surgically (392 cases) or endoscopically (29 cases) resected gastric adenocarcinoma patients with sAFP results before resection. Group B included 12 non-resected gastric adenocarcinoma patients with serum $\mathrm{AFP} \geq 7 \mathrm{ng} / \mathrm{mL}$ before treatment (group B).

\section{Clinicopathological characteristics and follow-up}

The clinicopathological information collected from all patients included sex, age, concurrent metastases, primary SAFP levels, Lauren classification and detailed histological classification (based on the Japanese Gastric Cancer Association classification [12]: tub1=well differentiated tubular type, tub2 $=$ moderately differentiated tubular type, por $1=$ solid type, por $2=$ poorly cohesive type, sig=signet-ring cell type, muc = mucin type, and other types). For group A, we also collected macroscopic classification, tumor location, tumor and node categories (American Joint Committee on Cancer (AJCC) 8th edition [13]), lymphovascular invasion (LVI), and perineural spread (PNS). Follow-up information included the location of postoperative recurrence in group A cases and the location of tumor progression in group B cases. The day of last follow-up was October 1, 2020.

\section{Serum AFP test}

Serum AFP was measured by an electrochemiluminescence assay using a Cobas e602 immunoassay analyzer (Roche Diagnostics, Germany) and the Elecsys AFP Kit (Roche Diagnostics, Germany). According to sAFP level before resection, cases in group A were divided into two groups: serum AFP-high (sAFP-H, $\quad$ sAFP $\geq 7 \mathrm{ng} / \mathrm{mL}$ ) and serum AFP-normal (sAFP-N, sAFP $<7 \mathrm{ng} / \mathrm{mL}$ ). The sAFP-H variation before and after resection was also recorded.

\section{Immunohistochemical test}

Immunohistochemical staining was performed with primary antibodies against AFP (1:100, Clone 1E4; Gene Tech Company Limited, Shanghai, China), GPC3 (1:100, Clone 1G12, ZSGB-Bio Company Limited, Beijing, China), and SALL4 (1:100, Clone 6E3, ZSGB-Bio Company Limited, Beijing, China). All immunohistochemical staining was performed in Ventana Benchmark XT. The results were considered positive when $\geq 1 \%$ cytoplasmic staining for AFP and GPC3 and $\geq 10 \%$ nuclear staining for SALL4 [14-16]. All standard and measured histopathological variables and all immunohistochemistry slides were first reviewed and graded independently by two authors (BW and LX), and then discordant cases 
were reviewed jointly until a consensus was reached. We divided the group A cases by the AFP/GPC3/SALL4 results into gastric cancer with primitive enterocyte phenotype (GAPEP, at least one of three was positive) and non-GAPEP (nGAPEP, all three markers were negative).

\section{Statistical analysis}

The clinicopathological characteristics and prognoses in groups A and B were analyzed. We performed 1:1 propensity score matching (PSM) in group A (both sAFP$\mathrm{N}$ vs. SAFP-H and GAPEP vs. nGAPEP) to compare the Progression Free Survival (PFS), and the PSM predictors included age, Lauren classification, and $\mathrm{T}$ and $\mathrm{N}$ categories (match tolerance $<0.2$, random seed to verify the reliability). Metastases in group B, including simultaneous metastases and metachronous metastases, were analyzed. The heatmap of A/G/S-IHC of sAFP-H cases was drawn by Prism 8 software (GraphPad Software Ltd., San Diego, USA). The relationship between immunohistochemistry and a high risk of disease progression (simultaneous metastases or postoperative metastases within three years) was analyzed. The comparison of count data was made by the chi-square test with a significance level of 0.05 on two-tailed P-values. SPSS (Statistical Product and Service Solutions, IBM Corp., NY, United States) 25.0 software was used for statistical analysis. Receiver operating characteristic (ROC) curves were plotted, and the area under the curve (AUC) and z-test were calculated.

\section{Results}

\section{Clinicopathological characteristics in group $\mathrm{A}$}

According to sAFP level, 404 (96.19\%) cases were assigned to the SAFP-N group, and 17 (3.81\%) cases were assigned to the SAFP-H group (Table 1). When the cases were divided by a cutoff age of 50 years, significantly more patients aged $<50$ years were noted in the sAFP-H group than those in the sAFP-N group $(P=0.023)$. Definite differences in both Lauren classification $(P=0.030)$ and JCGA histological classification $(P=0.021)$ were identified. Regarding the JGCA classification, the sAFP$\mathrm{H}$ cases mainly composed of tub2 type $(8,47.06 \%)$ and por1 type $(4,23.53 \%)$. More por2 type were observed in the sAFP-N cases. Notably, few cases of special subtypes were noted in the SAFP-H group: only 2 cases were HAC, 1 case was GAED, and the other cases were mostly moderately or poorly differentiated tubular adenocarcinoma with no special morphological features (Fig. 1). There were significant differences in $\mathrm{T}$ categories $(P=0.014)$. More T4 cases were in the SAFP-H cases $(10,58.82 \%)$ than those in the sAFP-N cases $(150,37.13 \%)$. Significant difference in $\mathrm{N}$ categories was identified $(P=0.047)$. The sAFP-H group had significantly more N3 cases than the sAFP-N group $(52.94 \%$ vs $29.60 \%)$. LVI $(76.47 \%$ vs
$35.15 \%, P<0.001)$ and PNS $(64.71 \%$ vs $33.42 \%, P=0.008)$ were significantly higher in the sAFP-H group than that in the sAFP-N group, respectively. The SAFP-H group had significantly more postoperative recurrence or metastases than the sAFP-N group $(23.53 \%$ vs $5.66 \%$, $P<0.001)$. Among the sAFP- $\mathrm{H}$ group, 3 patients had liver metastasis, and 1 patient had recurrence of the remnant stomach at one year after surgery. After 1:1 PSM, sAFP$\mathrm{H}$ patients also had worse PFS than sAFP-N patients $(P=0.048)$ (Fig. 2A). There were no significant differences in sex $(P=0.779)$, location $(P=0.164)$, or macroscopic classification $(P=0.064)$.

By the A/G/S-IHC results, 34/421 (8.08\%) cases were GAPEP. The positive rate of AFP was $4.28 \%$ (18/421), the positive rate of GPC3 was $4.51 \%(19 / 421)$, and the positive rate of SALL 4 was $2.85 \%$ (12/421). The GAPEP cases also showed similar characteristics as sAFP-H cases, especially in JGCA classification $(P=0.009)$ and T category $(P=0.001)$. The GAPEP group had significantly more postoperative recurrence or metastases than the nGAPEP group $(23.53 \%$ vs $2.07 \%, P<0.001) 1$ year after surgery. After 1:1 PSM, GAPEP patients also had worse survival than nGAPEP patients $(P=0.035)$ (Fig. 2B).

Serum AFP was re-examined in 7 sAFP-H patients after surgery. The sAFP levels in these 7 patients were significantly decreased after surgery, and most fell in the normal range within one year after surgery (Fig. 3A).

\section{Clinicopathological characteristics in group $B$}

In group B, a total of 7 cases had simultaneous metastases (58.33\%), of which 4 cases were liver metastases, 2 cases were ovarian metastases, and 1 case was liver metastases accompanied by lung metastases at the same time. The rate of liver metastases reached $41.67 \%$. Two patients had metachronous liver metastases during follow-up. The total liver metastasis rate reached $58.33 \%$. For the A/G/SIHC results, 11/12 cases were GAPEP.

The clinicopathological characteristics of the 29 sAFP-H cases in groups A and B are shown in Table 2. Interestingly, all patients with $\mathrm{sAFP}>1000 \mathrm{ng} / \mathrm{mL}$ in groups A and B had liver metastases, most of whom had liver metastases at diagnosis, while only one case had metachronous metastasis (Fig. 3B).

\section{Comparison and correlation of immunohistochemistry and sAFP test}

Serum AFP-H patients had significantly higher positive rates of AFP, GPC3, and SALL4 than SAFP-N patients (Table 3). Serum AFP-H was significantly related to GAPEP $(P<0.001)$. Notably, more high-risk cases were detected by immunohistochemistry, 17 surgical cases of GAPEP with normal sAFP were identified, 4 of which had postoperative recurrence or metastasis (2 
Table 1 Comparison of clinicopathological characteristics between sAFP-N vs. sAFP-H and between nGAPEP vs. GAPEP in the surgical cases (group A)

\begin{tabular}{|c|c|c|c|c|c|c|c|}
\hline & & SAFP-N & SAFP-H & $P$ & nGAPEP & GAPEP & $P$ \\
\hline \multirow[t]{2}{*}{ Age } & $\leq 50$ & 93 (23.02\%) & $8(47.06 \%)$ & 0.023 & 91 (23.51\%) & 10 (29.41\%) & 0.440 \\
\hline & $>50$ & $311(76.98 \%)$ & $9(52.94 \%)$ & & $296(76.49 \%)$ & 24 (70.59\%) & \\
\hline \multirow[t]{2}{*}{ Sex } & Male & $272(67.33 \%)$ & $12(70.59 \%)$ & 0.779 & $259(66.93 \%)$ & $25(73.53 \%)$ & 0.431 \\
\hline & Female & $132(32.67 \%)$ & $5(29.31 \%)$ & & $128(33.07 \%)$ & $9(26.47 \%)$ & \\
\hline \multirow[t]{3}{*}{ Location $^{*}$} & Proximal & $64(16.04 \%)$ & $2(11.76 \%)$ & 0.164 & $60(15.67 \%)$ & $6(18.18 \%)$ & 0.359 \\
\hline & Distal & 275 (68.92\%) & 15 (88.24\%) & & $265(69.19 \%)$ & 25 (75.76\%) & \\
\hline & Overlapping & 60 (15.04\%) & $0(0.00 \%)$ & & $58(15.14 \%)$ & $2(6.06 \%)$ & \\
\hline \multirow[t]{3}{*}{ Lauren classification } & Intestinal & $88(21.78 \%)$ & $8(47.06 \%)$ & 0.030 & $83(21.45 \%)$ & $13(38.24 \%)$ & 0.081 \\
\hline & Diffuse & $262(64.85 \%)$ & $6(35.29 \%)$ & & $251(64.86 \%)$ & $17(50.00 \%)$ & \\
\hline & Mixed & $54(13.37 \%)$ & $3(17.65 \%)$ & & $53(13.69 \%)$ & $4(11.76 \%)$ & \\
\hline \multirow[t]{7}{*}{ Histological classification ${ }^{* *}$} & Tub1 & $10(2.48 \%)$ & $0(0.00 \%)$ & 0.021 & $10(2.58 \%)$ & $0(0 \%)$ & 0.009 \\
\hline & Tub2 & $72(17.82 \%)$ & $8(47.06 \%)$ & & $68(17.57 \%)$ & $12(35.29 \%)$ & \\
\hline & Muc & $6(1.49 \%)$ & $0(0.00 \%)$ & & $5(1.29 \%)$ & $1(2.94 \%)$ & \\
\hline & Sig & $139(34.41 \%)$ & $0(0.00 \%)$ & & 137 (35.40\%) & $2(5.88 \%)$ & \\
\hline & Por1 & $62(15.35 \%)$ & $4(23.53 \%)$ & & $58(14.99 \%)$ & $8(23.53 \%)$ & \\
\hline & Por2 & $61(15.10 \%)$ & $2(11.76 \%)$ & & 56 (14.47\%) & 7 (20.59\%) & \\
\hline & Others & $54(13.37 \%)$ & $3(17.65 \%)$ & & $53(13.70 \%)$ & $4(11.76 \%)$ & \\
\hline \multirow[t]{4}{*}{ T category } & $\mathrm{T} 1$ & $142(35.15 \%)$ & $0(0.00 \%)$ & 0.014 & $140(36.18 \%)$ & $2(5.88 \%)$ & 0.001 \\
\hline & $\mathrm{T} 2$ & $57(14.11 \%)$ & $5(29.41 \%)$ & & $52(13.44 \%)$ & 10 (29.41\%) & \\
\hline & T3 & 55 (13.61\%) & $2(11.76 \%)$ & & 54 (13.95\%) & $3(8.82 \%)$ & \\
\hline & $\mathrm{T} 4$ & $150(37.13 \%)$ & 10 (58.82\%) & & $141(36.43 \%)$ & 19 (55.88\%) & \\
\hline \multirow[t]{4}{*}{ N category } & No & $150(40.00 \%)$ & $3(17.65 \%)$ & 0.047 & 141 (39.39\%) & 12 (35.29\%) & 0.152 \\
\hline & N1 & $63(16.80 \%)$ & $1(5.88 \%)$ & & $62(17.32 \%)$ & $2(5.88 \%)$ & \\
\hline & N2 & 51 (13.60\%) & $4(23.53 \%)$ & & $47(13.13 \%)$ & 8 (23.53\%) & \\
\hline & N3 & 111 (29.60\%) & $9(52.94 \%)$ & & $108(30.17 \%)$ & 12 (35.29\%) & \\
\hline \multirow[t]{2}{*}{ LVI } & No & $262(64.85 \%)$ & $4(23.53 \%)$ & 0.001 & 249 (64.34\%) & 17 (50.00\%) & 0.096 \\
\hline & Yes & $142(35.15 \%)$ & 13 (76.47\%) & & $138(35.66 \%)$ & 17 (50.00\%) & \\
\hline \multirow[t]{2}{*}{ PNS } & No & $269(66.58 \%)$ & $6(35.29 \%)$ & 0.008 & $257(66.41 \%)$ & 18 (52.94\%) & 0.114 \\
\hline & Yes & 135 (33.42\%) & $11(64.71 \%)$ & & $130(33.59 \%)$ & $16(47.06 \%)$ & \\
\hline \multirow[t]{2}{*}{ Postoperative recurrence/metastasis } & No & $200(94.34 \%)$ & $13(76.47 \%)$ & $<0.001$ & $379(97.93 \%)$ & $26(76.47 \%)$ & $<0.001$ \\
\hline & Yes & $12(5.66 \%)$ & $4(23.53 \%)$ & & $8(2.07 \%)$ & $8(23.53 \%)$ & \\
\hline
\end{tabular}

LVI, lymphovascular invasion; PNS, perineural spread

*Proximal=tumor in upper $1 / 2$ with a proximal gastrectomy; dismal=tumor in lower $1 / 2$ with a dismal gastrectomy; overlapping=large tumor or linitis plastica with a total gastrectomy

**Based on the Japanese Gastric Cancer Association classification, tub1 = well differentiated tubular type, tub2 = moderately differentiated tubular type, por $1=$ solid type, por $2=$ poorly cohesive type, sig = signet-ring cell type, muc=mucin type, and other types

cases of recurrence in gastric remnants, 1 case of liver metastasis, and 1 case of lung metastasis).

With a high risk of disease progression as the observation index, both methods had good efficacy (AUC $\mathrm{sAFP}=0.625, \mathrm{AUC}_{\mathrm{A} / \mathrm{G} / \mathrm{S}-\mathrm{IHC}}=0.723, \mathrm{z}$ statistic $=1.726$, $P=0.084$ ) (Fig. 4). The serum test had higher specificity $(100 \%$ vs $94.5 \%)$ while the immunohistochemical test had higher sensitivity ( $50 \%$ vs $25 \%$ ). We also drew a heatmap of all sAFP-H cases (Fig. 5).

\section{Discussion}

In this study, we compared the serum AFP test and immunohistochemical test of GAPEP to find the value of the two methods in clinical diagnosis. The proportion of sAFP-H patients in group A was $1.82 \%$, which was slightly lower than the number reported in the literature [17]. The reason might be this study is a retrospective research and not every patient underwent the sAFP test in our hospital. 


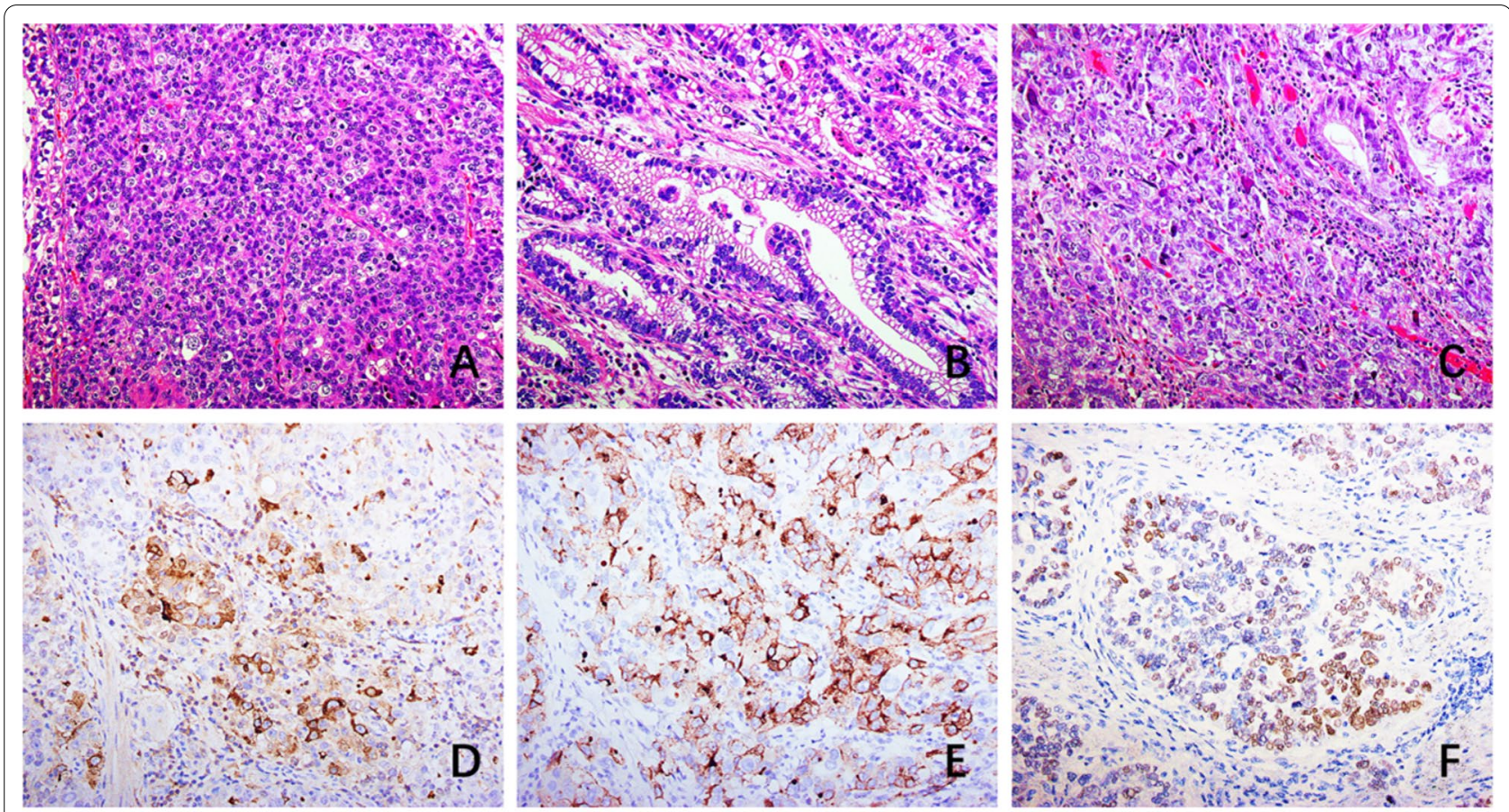

Fig. 1 A Hepatoid carcinoma area. B Adenocarcinoma with enteroblastic differentiation. C Moderately poorly differentiated adenocarcinomas with positive AFP (D), GPC3 (E) and SALL4 (F). All X200

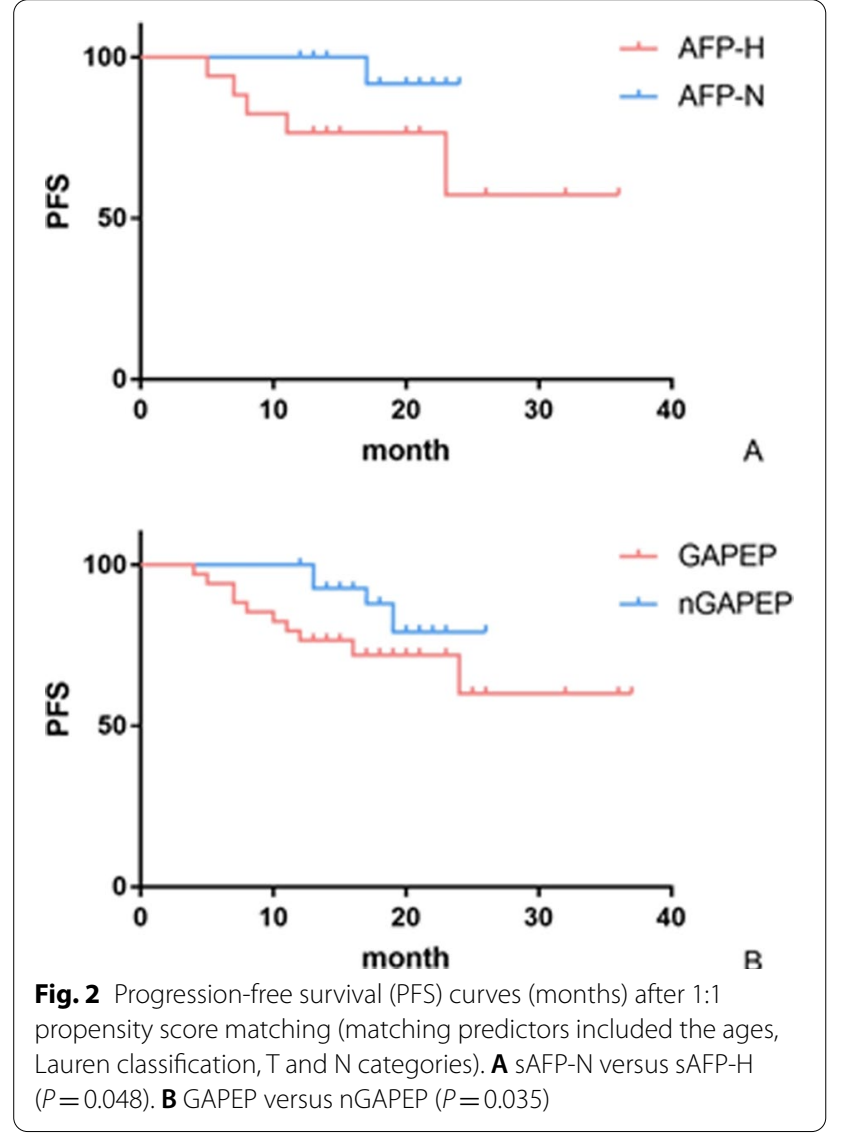

Though some studies argued the predictive value of serum AFP [18], most previous studies have confirmed that the prognosis of even early gastric adenocarcinoma with elevated serum AFP is poor [19]. It is believed that the production of sAFP is more likely to be caused by gastric cancer itself. AFP produced by the tumor itself that leads to an increase in the serum, and sAFP loses its source after tumor resection and then rapidly decreases, similar to our result (Fig. 3A). Some cases (3/8) with sAFP dropping after surgery had progression later, which might reflect the aggressive characteristics. However, the quantity of cases with both pre- and post-operation sAFP was limited. Another phenomenon that must be noted is that patients with extremely high sAFP values (>1000 ng/ $\mathrm{mL}$ ) had liver metastases at diagnosis, which may reflect high tumor burden. It is suggested that the sAFP test could be an important signal of liver metastasis. This study did not include biopsy cases with normal sAFP as a control for group B. Serum AFP-H patients often have simultaneous metastases at diagnosis, which is sufficient to demonstrate the biological characteristics. Further controlled studies of SAFP-H and sAFP-N in biopsy cases are necessary to better understand the implication of sAFP-H.

The mechanism of the special biological behavior of liver metastasis has been explored in some studies [20, 21]. One reason may be that this tumor produces many 

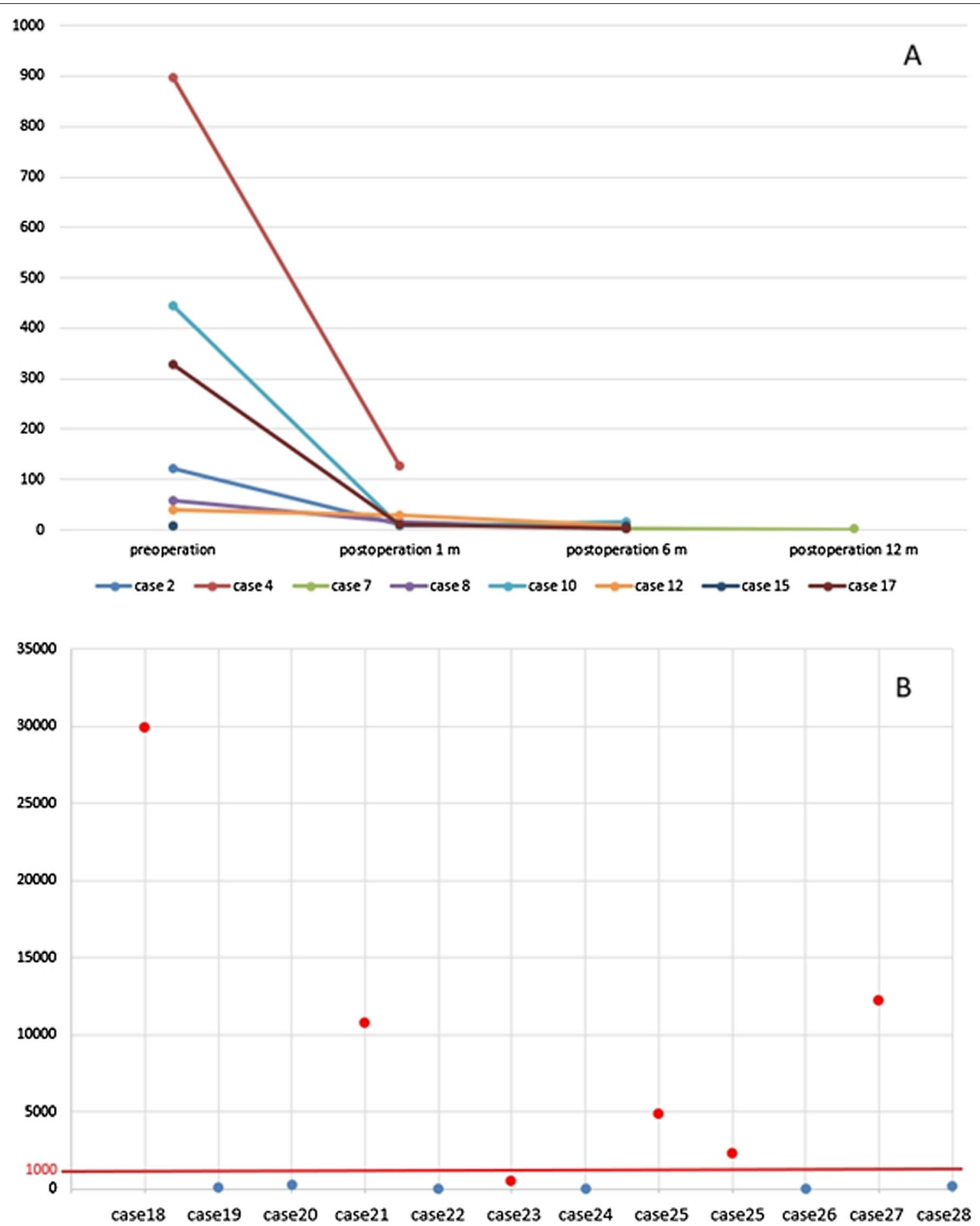

Fig. 3 A The serum AFP value $(\mathrm{ng} / \mathrm{mL})$ varied from the preoperative period to the postoperative period in 8 cases in group A. B The serum AFP value $(\mathrm{ng} / \mathrm{mL})$ of 12 cases in group B. Each dot represents a case, and the red dot indicates liver metastases

tumor thrombi, and the liver and lungs are vital for blood circulation, so the tumor thrombi easily spread to the liver through the blood [22]. Another possible reason is that the tumor itself activates certain molecular pathways, so it easily colonizes and grows in the liver [23, 24]. From the perspective of embryonic development, the liver, gastrointestinal tract, and lung originate from the endoderm, and those organs develop closely in 





Table 3 Correlation between serum AFP value and immunohistochemical results

\begin{tabular}{lllll}
\hline & & SAFP-N & SAFP-H & $P$ \\
\hline AFP & $(-)$ & $403(99.75 \%)$ & 0 & $<0.001$ \\
& $(+)$ & $1(0.25 \%)$ & $17(100.00 \%)$ & \\
GPC3 & $(-)$ & $392(97.03 \%)$ & $10(58.82 \%)$ & $<0.001$ \\
& $(+)$ & $12(2.97 \%)$ & $7(41.18 \%)$ & \\
SALL4 & $(-)$ & $399(98.76 \%)$ & $10(58.82 \%)$ & $<0.001$ \\
& $(+)$ & $5(1.24 \%)$ & $7(41.18 \%)$ & \\
GAPEP & $(-)$ & $387(95.79 \%)$ & 0 & $<0.001$ \\
& $(+)$ & $17(4.21 \%)$ & $17(100 \%)$ & \\
\hline
\end{tabular}

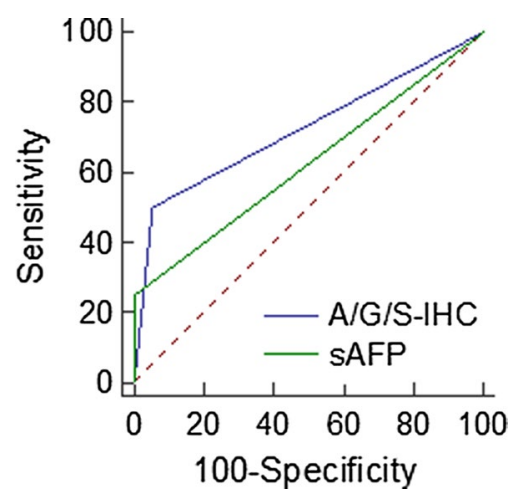

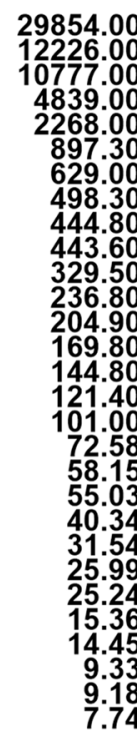

\section{AFP}

GPC3

Fig. 5 Heatmap of all SAFP-H cases in groups A and B. Horizontal axis, AFP, GPC3 and SALL4 results. Vertical axis, SAFP-H cases in descending order of SAFP level

of SMAD4 heterozygosity by immunohistochemistry in gastric adenocarcinoma with enteroblastic differentiation $[33,34]$. Yoichi et al. found that GAED frequently harbors TP53 mutations and ERBB2 amplification [35]. Fujimoto et al. found that HER2 is frequently overexpressed in hepatoid adenocarcinoma and gastric carcinoma with enteroblastic differentiation [36]. According to our previous research, GAPEP accounted for $7.53 \%$ of surgical specimens [37]. Defining this particular type may have some significance in selecting patients for more aggressive treatment [38]. However, several large-scale gastric cancer molecular typing studies, such as The Cancer Genome Atlas (TCGA) and the Asian Cancer Research Group (ACRG) [39-42], have not included GAPEP. More studies are needed on the molecular pathological features of GAPEP.

There were still some weaknesses in this research. GA with AFP-producing and GAPEP were rare tumor, and the quantity of those cases was limited. More evidences are in need to confirm the results in future. And we couldn't establish the comparator arm in group B because the complex reasons for not getting operation. The two methods both have their own advantages, the serological method is noninvasive and specific. However, the results may be affected by other factors (liver disease, germ cell tumors, etc.). Although immunohistochemical examination is invasive, it is sensible and can better reflect the nature of the tumor. In applying both methods to determine the lar research on GAPEP and its related tumors has yielded some results [31, 32]. Noboru et al. found frequent loss 
prognosis of a patient, the AUC was slightly better than using only one of the two. Therefore, we recommend using sAFP and AFP/GPC3/SALL4 immunohistochemistry for all cases of gastric cancer.

\section{Conclusions}

Both the serum AFP level and immunohistochemical expression of can be used to indicate a poor prognosis for gastric adenocarcinoma. The serum AFP level (especially $>1000 \mathrm{ng} / \mathrm{mL}$ ) is more specific, and immunohistochemistry is more sensitive.

\section{Acknowledgements}

None.

\section{Authors' contributions}

LX and YX performed study concept and design; BW, LZ and XZ performed development of methodology and writing, review and revision of the paper; $\mathrm{NL}, J \mathrm{G}$ and $\mathrm{ZL}$ provided acquisition, analysis and interpretation of data, and statistical analysis; YY and XL provided technical and material support. All authors read and approved the final paper.

\section{Funding}

This work was supported by the Beijing Hope Run Special Fund (No.

LC2018A12), the CAMS Initiative for Innovative Medicine (CIFMS) (No. 2016-

I2M-3-005), the Medical and Health Science and Technology Innovation

Project of the Chinese Academy of Medical Sciences (2016-12M-1-007), and the China International Medical Exchange Foundation Xiansheng Anti-Tumor Therapy Special Research Fund (cimf-f-h001-314).

\section{Availability of data and materials}

The datasets used and/or analysed during the current study available from the corresponding author on reasonable request.

\section{Declarations}

\section{Ethics approval and consent to participate}

Ethical approval was approved by CICAMS ethics committee prior to commencing this study (No. 21/105-2776). All methods were carried out in accordance with relevant guidelines and regulations, and informed consent was obtained from all participants.

\section{Consent for publication}

Not applicable.

\section{Competing interests}

The authors declare that they have no competing interests.

\begin{abstract}
Author details
${ }^{1}$ Department of Pathology, National Cancer Center/National Clinical Research Center for Cancer/Cancer Hospital, Chinese Academy of Medical Sciences and Peking Union Medical College, Beijing 100021, China. ${ }^{2}$ Department of Abdominal Surgical Oncology, National Cancer Center/National Clinical Research Center for Cancer/Cancer Hospital, Chinese Academy of Medical Sciences and Peking Union Medical College, Beijing 100021, China. ${ }^{3}$ Department of General Surgery, the First People's Hospital of Dongcheng District, Beijing 100075, China. ${ }^{4}$ Department of Clinical Laboratory, National Cancer Center/National Clinical Research Center for Cancer/Cancer Hospital, Chinese Academy of Medical Sciences and Peking Union Medical College, Beijing 100021, China.
\end{abstract}

Received: 6 April 2021 Accepted: 18 October 2021

Published online: 27 October 2021

\section{References}

1. Torre LA, Siegel RL, Ward EM, Jemal A. Global cancer incidence and mortality rates and trends: an update. Cancer Epidemiol Biomarkers Prev. 2016;25(1):16-27.

2. He CZ, Zhang KH, Li Q, Liu XH, Hong Y, Lv NH. Combined use of AFP, CEA, CA125 and CAl9-9 improves the sensitivity for the diagnosis of gastric cancer. BMC Gastroenterol. 2013;13:87

3. Feng F, Tian Y, Xu G, Liu Z, Liu S, Zheng G, et al. Diagnostic and prognostic value of CEA, CA19-9, AFP and CA125 for early gastric cancer. BMC Cancer. 2017;17(1):737

4. Bourreille J, Metayer P, Sauger F, Matray F, Fondimare A. Existence of alpha feto protein during gastric-origin secondary cancer of the liver. Presse Med. 1970;78(28):1277-8.

5. Ishikura H, Fukasawa Y, Ogasawara K, Natori T, Tsukada Y, Aizawa M. An AFP-producing gastric carcinoma with features of hepatic differentiation: a case report. Cancer. 1985;56(4):840-8.

6. Lakshmanan A, Kurian A, Subramanyan A, Srinivasan A. An alpha fetoprotein producing gastric tumor with yolk sac, hepatoid and papillary adenocarcinoma components. J Clin Diagn Res. 2017;11(9):ED03-5.

7. Murakami T, Yao T, Mitomi H, Morimoto T, Ueyama H, Matsumoto K, et al. Clinicopathologic and immunohistochemical characteristics of gastric adenocarcinoma with enteroblastic differentiation: a study of 29 cases. Gastric Cancer. 2016;19(2):498-507.

8. Kinjo T, Taniguchi H, Kushima R, Sekine S, Oda I, Saka M, et al. Histologic and immunohistochemical analyses of alpha-fetoprotein-producing cancer of the stomach. Am J Surg Pathol. 2012;36(1):56-65.

9. Xie Y, Zhao Z, Li P, Wang Y, Guo C, Wang X, et al. Hepatoid adenocarcinoma of the stomach is a special and easily misdiagnosed or missed diagnosed subtype of gastric cancer with poor prognosis but curative for patients of pN0/1: the experience of a single center. Int J Clin Exp Med. 2015;8(5):6762-72.

10. Yamazawa S, Ushiku T, Shinozaki-Ushiku A, Hayashi A, Iwasaki A, Abe H, et al. Gastric cancer with primitive enterocyte phenotype: an aggressive subgroup of intestinal-type adenocarcinoma. Am J Surg Pathol. 2017:41(7):989-97.

11. Chang YC, Nagasue N, Abe S, Taniura H, Kumar DD, Nakamura T. Comparison between the clinicopathologic features of AFP-positive and AFP-negative gastric cancers. Am J Gastroenterol. 1992;87(3):321-5.

12. JGC Association. Japanese classification of gastric carcinoma. 15th ed. Tokyo: Kanehara \& Co., Ltd; 2017.

13. Cancer AJCO. AJCC cancer staging manual. 8th ed. Berlin: Springer; 2017.

14. Ushiku T, Shinozaki A, Shibahara J, Iwasaki Y, Tateishi Y, Funata N, et al. SALL4 represents fetal gut differentiation of gastric cancer, and is diagnostically useful in distinguishing hepatoid gastric carcinoma from hepatocellular carcinoma. Am J Surg Pathol. 2010;34(4):533-40.

15. Ushiku T, Uozaki H, Shinozaki A, Ota S, Matsuzaka K, Nomura S, et al. Glypican 3-expressing gastric carcinoma: distinct subgroup unifying hepatoid, clear-cell, and alpha-fetoprotein-producing gastric carcinomas. Cancer Sci. 2009;100(4):626-32.

16. Osada M, Aishima S, Hirahashi M, Takizawa N, Takahashi S, Nakamura K, et al. Combination of hepatocellular markers is useful for prognostication in gastric hepatoid adenocarcinoma. Hum Pathol. 2014;45(6):1243-50.

17. Su JS, Chen YT, Wang RC, Wu CY, Lee SW, Lee TY. Clinicopathological characteristics in the differential diagnosis of hepatoid adenocarcinoma: a literature review. World J Gastroenterol. 2013;19(3):321-7.

18. Hirajima S, Komatsu S, Ichikawa D, Kubota T, Okamoto K, Shiozaki A, et al. Liver metastasis is the only independent prognostic factor in AFP-producing gastric cancer. World J Gastroenterol. 2013;19(36):6055-61.

19. Yao M, Zhao J, Lu F. Alpha-fetoprotein still is a valuable diagnostic and prognosis predicting biomarker in hepatitis B virus infection-related hepatocellular carcinoma. Oncotarget. 2016;7(4):3702-8.

20. Vidal-Vanaclocha F, Crende O, Garcia de Durango C, Herreros-Pomares A, Lopez-Domenech S, Gonzalez A, et al. Liver prometastatic reaction: Stimulating factors and responsive cancer phenotypes. Semin Cancer Biol. 2021;71:122-33.

21. Clark AM, Allbritton NL, Wells A. Integrative microphysiological tissue systems of cancer metastasis to the liver. Semin Cancer Biol. 2021;71:157-69.

22. Chang YC, Nagasue N, Abe S, Kohno H, Kumar DD, Nakamura T. alpha Fetoprotein producing early gastric cancer with liver metastasis: report of three cases. Gut. 1991;32(5):542-5. 
23. Asahi Y, Kamiyama T, Homma S, Hatanaka KC, Yokoo H, Nakagawa T, et al. Resection of liver metastasis derived from alpha-fetoprotein-producing gastric cancer-report of 4 cases. Int Cancer Conf J. 2016;5(2):98-103.

24. Chun H, Kwon SJ. Clinicopathological characteristics of alpha-fetoproteinproducing gastric cancer. J Gastric Cancer. 2011;11(1):23-30.

25. Chen D, Lin X, Zhang C, An G, Li Z, Dong B, et al. Activated Wnt signaling promotes growth and progression of AFP-producing gastric cancer in preclinical models. Cancer Manag Res. 2019;11:1349-62.

26. Han L, Chaturvedi P, Kishimoto K, Koike H, Nasr T, Iwasawa K, et al. Single cell transcriptomics identifies a signaling network coordinating endoderm and mesoderm diversification during foregut organogenesis. Nat Commun. 2020;11(1):4158.

27. Mills SE. Histology for pathologists. Philadelphia: Lippincott Williams and Wilkins; 2012.

28. Furuya Y, Wakahara T, Akimoto H, Kishimoto T, Hiroshima $K$, Yanagie $H$, et al. Clear cell adenocarcinoma with enteroblastic differentiation of the ascending colon. J Clin Oncol. 2011;29(22):e647-649.

29. Mitsuma K, Taniguchi H, Kishi Y, Hiraoka N. A case of adenocarcinoma with enteroblastic differentiation of the ampulla of Vater. Pathol Int. 2016:66(4):230-5.

30. Ricaurte LM, Arrieta O, Zatarain-Barron ZL, Cardona AF. Comprehensive review of fetal adenocarcinoma of the lung. Lung Cancer (Auckl). 2018;9:57-63.

31. Arora K, Bal M, Shih A, Moy A, Zukerberg L, Brown I, et al. Fetal-type gastrointestinal adenocarcinoma: a morphologically distinct entity with unfavourable prognosis. J Clin Pathol. 2018;71(3):221-7.

32. Lu J, Ding Y, Chen Y, Jiang J, Chen Y, Huang Y, et al. Whole-exome sequencing of alpha-fetoprotein producing gastric carcinoma reveals genomic profile and therapeutic targets. Nat Commun. 2021;12(1):3946.

33. Li T, Liu T, Wang M, Zhang M. Alpha-fetoprotein producing hepatoid gastric adenocarcinoma with neuroendocrine differentiation: a case report. Medicine (Baltimore). 2018;97(37):e12359.

34. Suzuki A, Koide N, Kitazawa M, Mochizuka A, Ota H, Miyagawa S. Gastric composite tumor of alpha fetoprotein-producing carcinoma/hepatoid adenocarcinoma and endocrine carcinoma with reference to cellular phenotypes. Patholog Res Int. 2012;2012:201375.
35. Yatagai N, Saito T, Akazawa Y, Hayashi T, Yanai Y, Tsuyama S, et al. Frequent loss of heterozygosity of SMAD4 locus and prognostic impacts of SMAD4 immunohistochemistry in gastric adenocarcinoma with enteroblastic differentiation. Hum Pathol. 2019;88:18-26.

36. Akazawa Y, Saito T, Hayashi T, Yanai Y, Tsuyama S, Akaike K, et al. Nextgeneration sequencing analysis for gastric adenocarcinoma with enteroblastic differentiation: emphasis on the relationship with hepatoid adenocarcinoma. Hum Pathol. 2018;78:79-88.

37. Fujimoto M, Matsuzaki I, Nishino M, Iwahashi Y, Warigaya K, Kojima F, et al. HER2 is frequently overexpressed in hepatoid adenocarcinoma and gastric carcinoma with enteroblastic differentiation: a comparison of 35 cases to 334 gastric carcinomas of other histological types. J Clin Pathol. 2018;71(7):600-7.

38. Wang B. The clinicopathologic characteristics and immunochemistry classifications in gastric adenocarcinoma. Beijing: Chinese Academy of Medical Sciences and Peking Union Medical College; 2018.

39. Xiao C, Wu F, Jiang H, Teng L, Song F, Wang Q, et al. Hepatoid adenocarcinoma of the stomach: nine case reports and treatment outcomes. Oncol Lett. 2015;10(3):1605-9.

40. Arakawa Y, Tamura M, Aiba K, Morikawa K, Aizawa D, Ikegami M, et al. Significant response to ramucirumab monotherapy in chemotherapyresistant recurrent alpha-fetoprotein-producing gastric cancer: a case report. Oncol Lett. 2017;14(3):3039-42.

41. Li Z, Hou X, Chen J, Sun H, Mi Y, Sui Y, et al. Efficacy and safety of SOX chemotherapy with or without surgery in AFP-producing advanced gastric cancer. Oncol Lett. 2017;14(1):579-86.

42. Wang Y, Shen L, Lu M, Ji Z, Zhang X. Multimodality treatment including triplet regimen as first-line chemotherapy may improve prognosis of serum AFP-elevated gastric cancer with liver metastasis. Gastroenterol Res Pract. 2017;2017:5080361

\section{Publisher's Note}

Springer Nature remains neutral with regard to jurisdictional claims in published maps and institutional affiliations.
Ready to submit your research? Choose BMC and benefit from:

- fast, convenient online submission

- thorough peer review by experienced researchers in your field

- rapid publication on acceptance

- support for research data, including large and complex data types

- gold Open Access which fosters wider collaboration and increased citations

- maximum visibility for your research: over 100M website views per year

At BMC, research is always in progress.

Learn more biomedcentral.com/submissions 